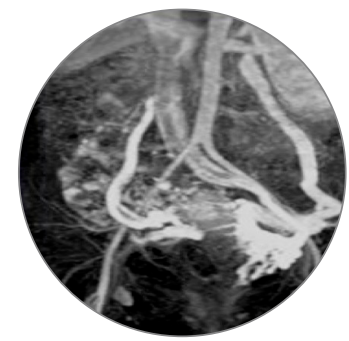

$\sum$

Palabras clave (DeCS)

Insuficiencia venosa

Dolor pélvico

Dispareunia

Key words (MeSH)

Venous insufficiency

Pelvic pain

Dyspareunia
'Médico radiólogo intervencionista, Hospital San Vicente Fundación. Profesor fellow de radiología intervencionista, Universidad de Antioquia. Medellín, Colombia.

Médico general, Universidad Pontificia Bolivariana. MedeIlín, Colombia.

Hospital San Vicente Fundación, Departamento de Radiología.

\title{
Estado del arte en el manejo endovascular del síndrome de congestión pélvica
}

\author{
State of the Art in the Endovascular Management of Pelvic \\ Congestion Syndrome
}

Luis Alberto Cruz Vásquez

Mateo Zapata Naranjo ${ }^{2}$

\section{Resumen}

El dolor pélvico crónico (DPC) se define como un dolor de localización pélvica con una duración mayor a 6 meses. Es una causa importante de consulta médica, y afecta entre el $24 \%$ y el $45 \%$ de las mujeres en el mundo. El síndrome de congestión pélvica (SPC) es una fuente notable y muchas veces poco diagnosticada de dolor pélvico crónico en pacientes, en su mayoría premenopáusicas, que afecta de forma relevante la calidad de vida. El principal objetivo de este artículo es demostrar las múltiples opciones diagnósticas y diferentes dispositivos terapéuticos que se usan para la embolización selectiva, para dar un adecuado manejo y resolución al síndrome de congestión pélvica. En conclusión, el SPC es una entidad altamente dolorosa, incapacitante e importante para tener en cuenta por su alta prevalencia y morbilidad asociadas. El tratamiento endovascular ha demostrado ser una opción altamente confiable, segura, con bajas tasas de complicaciones, menor tiempo de recuperación y estancias hospitalarias más cortas, en comparación con la opción quirúrgica. Es importante conocer el material y utilizarlo correctamente, lo cual ayudará a evitar complicaciones.

\section{Summary}

Chronic pelvic pain (CPP) is defined as pain in the pelvic area with a duration more than 6 months. It's an important cause of medical consultation, affecting approximately between 24 and $45 \%$ of women in the world. Pelvic congestion syndrome (PCS) is a notable and often under-diagnosed cause of chronic pelvic pain in patients, mostly premenopausal, that significantly affects quality of life. The main objective of this paper is to demonstrate the multiple diagnostic options and different therapeutic devices that are used for selective embolization, giving adequate management and resolution of pelvic congestion syndrome. In conclusion, PCS is a highly painful, disabling and important pathology to take into account due to its high prevalence and associated morbidity. Endovascular treatment has proven to be a highly reliable and safe option, with low complication rates, shorter recovery time, and briefer hospital stays compared to the surgical option. It's important to know the material and use it correctly which will help avoid complications.

\section{Introducción}

El dolor pélvico crónico (DPC) se define como un dolor de localización pélvica, con una duración mayor a 6 meses. Es una causa importante de consulta médica, que afecta entre el 24 y el $45 \%$ de las mujeres en el mundo (1,2), y el $15 \%$ de las mujeres entre los 18 y los 50 años de edad, en Estados Unidos (2); es responsable del 10 al $30 \%$ de las atenciones médicas ginecológicas (3-5) y genera un costo anual cercano a los 2 billones de dólares en Estados Unidos (6) y 3,8 billones de euros en Europa (7).

El síndrome de congestión pélvica (SPC) es una fuente notable $-\mathrm{y}$ muchas veces poco diagnosticada $(4,8)$ - de dolor pélvico crónico en pacientes, en su mayoría, premenopáusicas, a quienes les afecta de forma relevante la calidad de vida $(1,3,9)$. Aproximadamente, entre el 20 y el $40 \%$ de las consultas por dolor pélvico crónico se deben a $\operatorname{SPC}(4,8,10)$.

El SPC, como causante de dolor pélvico crónico, fue descrito por primera vez en 1949 por Howard Taylor (11). La sintomatología es secundaria al flujo retrógrado de las venas gonadales incompetentes, lo que genera dilatación venosa pélvica y congestión tisular asociada $(12,13)$. Además, cabe resaltar que hay factores mecánicos implicados en la patogénesis de la misma, como la ausencia de valvas venosas ováricas hasta en el $15 \%$ del lado izquierdo y $6 \%$ del lado derecho, e incompetencia valvular de al menos uno de los dos lados en el $50 \%$ de las pacientes $(12,14)$. El componente genético juega un papel importante en el desarrollo de la enfermedad, pues se ha identificado asociación hasta en el $50 \%$ de las pacientes. Igualmente, el influjo hormonal se considera 
un factor determinante de la patogénesis, específicamente en cuanto al estradiol, el cual induce dilatación selectiva de las venas ováricas y uterinas, lo que se traduce en un aumento en el estrés valvular $(5,8)$. Otro mecanismo fisiopatológico implicado es la obstrucción al flujo venoso ovárico, específicamente, la compresión de la vena renal izquierda por parte de la arteria mesentérica superior y la aorta, que desencadena en el síndrome de cascanueces (15).

Los principales factores de riesgo asociados a la enfermedad son: multiparidad, antecedente familiar de insuficiencia venosa, edad entre 20 y 45 años (secundario al mecanismo de influjo hormonal), antecedente de várices glúteas, vulvares y perineales, insuficiencia venosa, procedimientos quirúrgicos pélvicos y útero retroverso. Además, hay una relación no muy clara entre SPC y ovarios poliquísticos, aunque se ha encontrado en el 40 y $50 \%$ de las pacientes con insuficiencia venosa pélvica $(4,5,8)$.

Típicamente, es un dolor que se manifiesta como una "pesadez" a la altura de la pelvis, de predominio vespertino, que empeora al estar de pie, con la menstruación, los esfuerzos y el embarazo, y mejora con la posición supina. Los síntomas, que en la mayoría de ocasiones acompañan al dolor, son dispareunia, disuria, dismenorrea, aumento en la frecuencia urinaria, urgencia urinaria, náuseas y várices visibles sintomáticas en la región vulvar y perineal, así como en las caras interna y posterior de los muslos $(3-5,9,16)$. La asociación con insuficiencia venosa de miembros inferiores es significativa, cercana al $15 \%$ (17).

\section{Diagnóstico}

$\mathrm{Al}$ examen físico en el tracto vaginal se puede encontrar sensibilidad a la movilización cervical, y en la palpación bimanual puede aparecer sensibilidad uterina y ovárica; además, se pueden visualizar venas varicosas vulvares y en los muslos. La combinación entre sensibilidad ovárica y dolor después de la relación sexual tiene una sensibilidad del $94 \%$ y una especificidad del $77 \%$ para SPC, cuando se evalúa posteriormente con venografía $(10,18,19)$.

Para establecer el diagnóstico es importante realizar una historia clínica completa, teniendo en cuenta los factores de riesgo mencionados, la caracterización del dolor y un adecuado examen físico. Adicional a esto, las ayudas imagenológicas son esenciales ya que permiten confirmarlo de forma definitiva; las principales herramientas que ayudan a establecer el diagnóstico son: ecografía transvaginal (con aplicación Doppler), ecografía transabdominal, venografía convencional, resonancia magnética $(\mathrm{RM})$ y tomografía computarizada (TAC) $(13,20)$.

\section{Imagenología}

La ecografía transvaginal con Doppler es el estudio ideal para el diagnóstico de SPC, debido a su naturaleza no invasiva, de bajo costo, sin radiación y altamente disponible; además, se puede realizar en posición semivertical y evaluar con maniobras de Valsalva, las cuales son obligatorias. Por otra parte, tiene la ventaja de que sumada a la ecografía transabdominal, permite excluir otros diagnósticos como causantes de la sintomatología. Como única limitación está el ser dependiente del operador. Los criterios diagnósticos con ecografía transvaginal son: várices parauterinas dilatadas múltiples, diámetro $>4 \mathrm{~mm}$, flujo lento $<=3 \mathrm{~cm} / \mathrm{seg}$, vena arcuata dilatada en el miometrio que cruza la línea media y configuración poliquística de los ovarios.
La ecografía transabdominal también es una imagen por tener en cuenta para el diagnóstico; los criterios son: flujo retrógrado en la vena gonadal derecha o izquierda dilatada, vena gonadal dilatada $>5$ $\mathrm{mm}$. La medición de venas ováricas mayores de $5 \mathrm{~mm}$ tiene un valor predictivo positivo (VPP) de $71 \%$ y mayores de $6 \mathrm{~mm}$, un VPP del $83 \%$ para el diagnóstico de $\operatorname{SCP}(5,9,20,21)$. En la figura 1 se puede observar una imagen por ecografía con estudio Doppler.

La venografía convencional con cateterización selectiva de las venas hipogástricas y gonadales es el estándar de oro para el diagnóstico de SCP $(3-5,9,20,22)$ (figuras 2 y 3$)$. Su principal ventaja radica en poder realizar el manejo endovascular de forma concomitante. Dentro de sus desventajas están la radiación y el hecho de ser invasiva. Los criterios diagnósticos son: arcadas venosas gonadales, uterinas y útero-ováricas dilatadas, diámetro $>5 \mathrm{~mm}$, flujo caudal retrógrado en la vena gonadal (unilateral o bilateral), llenado de las venas pélvicas pasando la línea media a través de la arcada útero-ovárica, opacificación de las várices vulvovaginales o de los muslos, remansamiento del medio de contraste en las venas pélvicas (5).

Es importante señalar que se deben cateterizar ambas venas hipogástricas y gonadales, así como realizar el estudio con maniobras de Valsalva e idealmente en mesa basculante. El abordaje se puede hacer vía vena basílica, vena yugular o vena femoral (9).

Por su parte, las imágenes por RM tienen utilidad importante en el diagnóstico, principalmente debido a su ausencia de radiación ionizante y ofrecen gran detalle de la anatomía y su funcionalidad, para descartar otros diagnósticos. Sus limitaciones se centran en su poca disponibilidad, alto costo y baja especificidad (9). La angiografía por resonancia magnética con resolución temporal (TR-MRA) es una variante con alto rendimiento demostrado por varios estudios; particularmente, en un ensayo publicado en 2012 en el cual se evaluaron 19 pacientes por venografía convencional y TR-MRA por parte de 2 observadores calificados, se obtuvo para esta última una sensibilidad entre 67 y $75 \%$, una especificidad del $100 \%$ y una precisión entre 79 y $84 \%$ (23). Los criterios diagnósticos incluyen: flujo caudal retrógrado del medio de contraste en la TR-MRA, várices parauterinas dilatadas, evidencia de lentitud en el flujo (heterogéneo o hiperintenso en T2), presencia de vena arcuata que cruza la línea media, várices vulvares o en los muslos, configuración poliquística de los ovarios, ausencia de obstrucción estructural o masa obstructora y sin evidencia de endometriosis $(5,23,24)$ (figura 4$)$.

Por último, la venografía por TAC tiene un papel mínimo en el abordaje diagnóstico del SCP. Se debe realizar cuando se sospeche algún tipo de anormalidad estructural; tiene utilidad en la caracterización anatómica previa a procedimientos y en la exclusión de diagnósticos diferenciales. Las venas pélvicas incompetentes se visualizan como estructuras tubulares ensanchadas, dilatadas y tortuosas alrededor de útero y ovarios. Sus desventajas están relacionadas con la radiación, su baja especificidad, la forma supina para su realización y, al igual que la RM, no permite determinar el reflujo del medio de contraste dentro de esas venas $(4,5,9)$. Los criterios diagnósticos propuestos son: 4 venas parauterinas ipsilaterales tortuosas y dilatadas (mínimo una mayor a $4 \mathrm{~mm}$ ), vena gonadal dilatada con un diámetro mayor a $8 \mathrm{~mm}$ unilateral o bilateral, ausencia de compresión estructural por alteración anatómica o masa y sin evidencia de endometriosis $(5,24)$ (figura 5). 


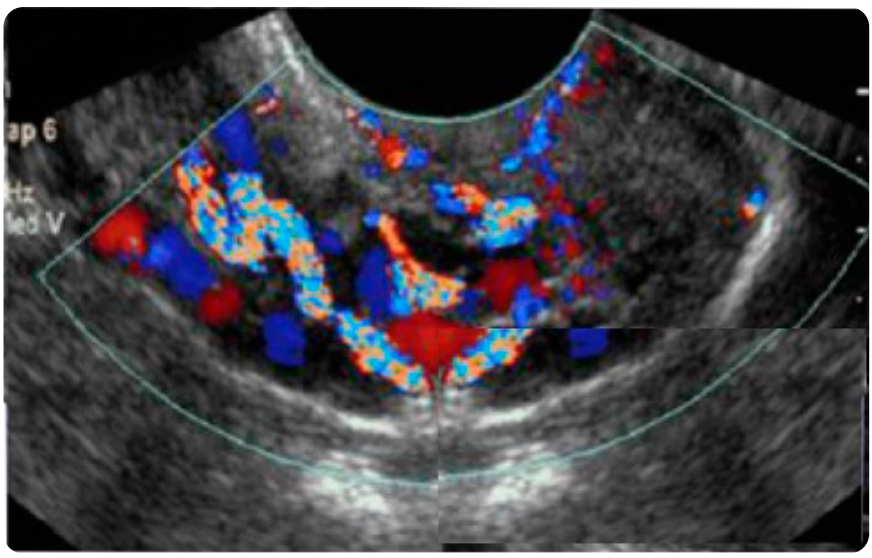

Figura 1. Ultrasonografía vaginal con análisis Doppler. Se observa dilatación del plejo venoso parauterino secundario a várices pélvicas

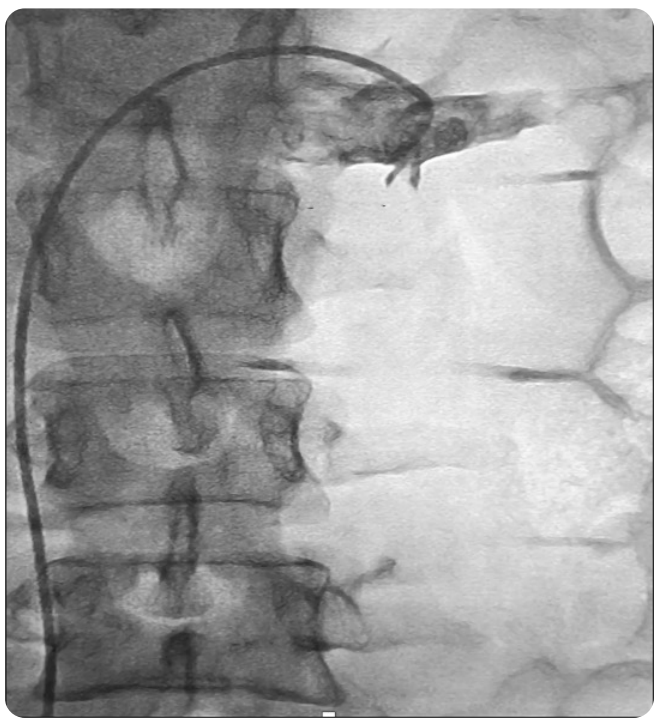

Figura 2. Venografía selectiva en la vena renal izquierda, que demuestra competencia de la vena gonadal izquierda y su válvula normal.

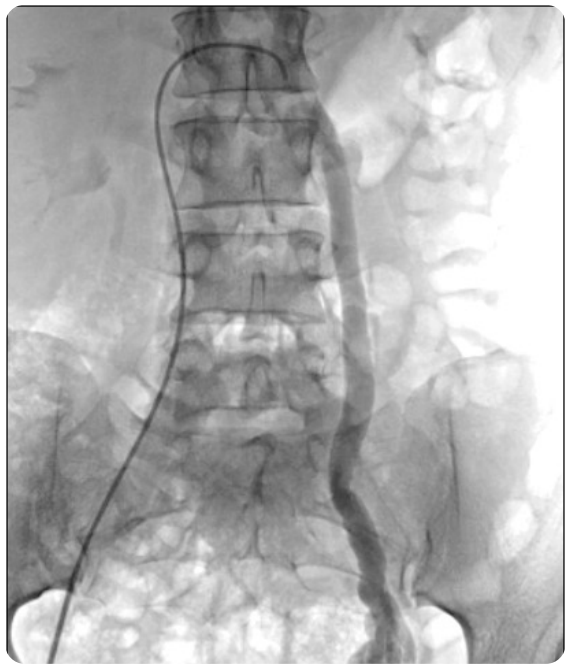

Figura 3. Venografía selectiva donde se evidencia la vena gonadal izquierda insuficiente.

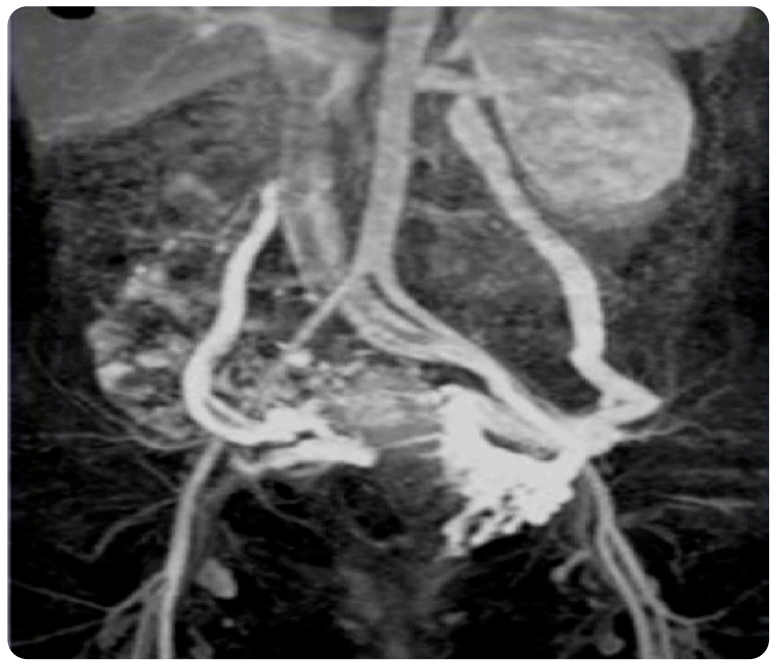

Figura 4. Venografía por RM. Se visualizan ambas venas gonadales con medio de contraste en su interior con evidencia clara de insuficiencia vascular venosa pélvica producida por dilatación de ambas venas gonadales y formación de várices pélvicas.
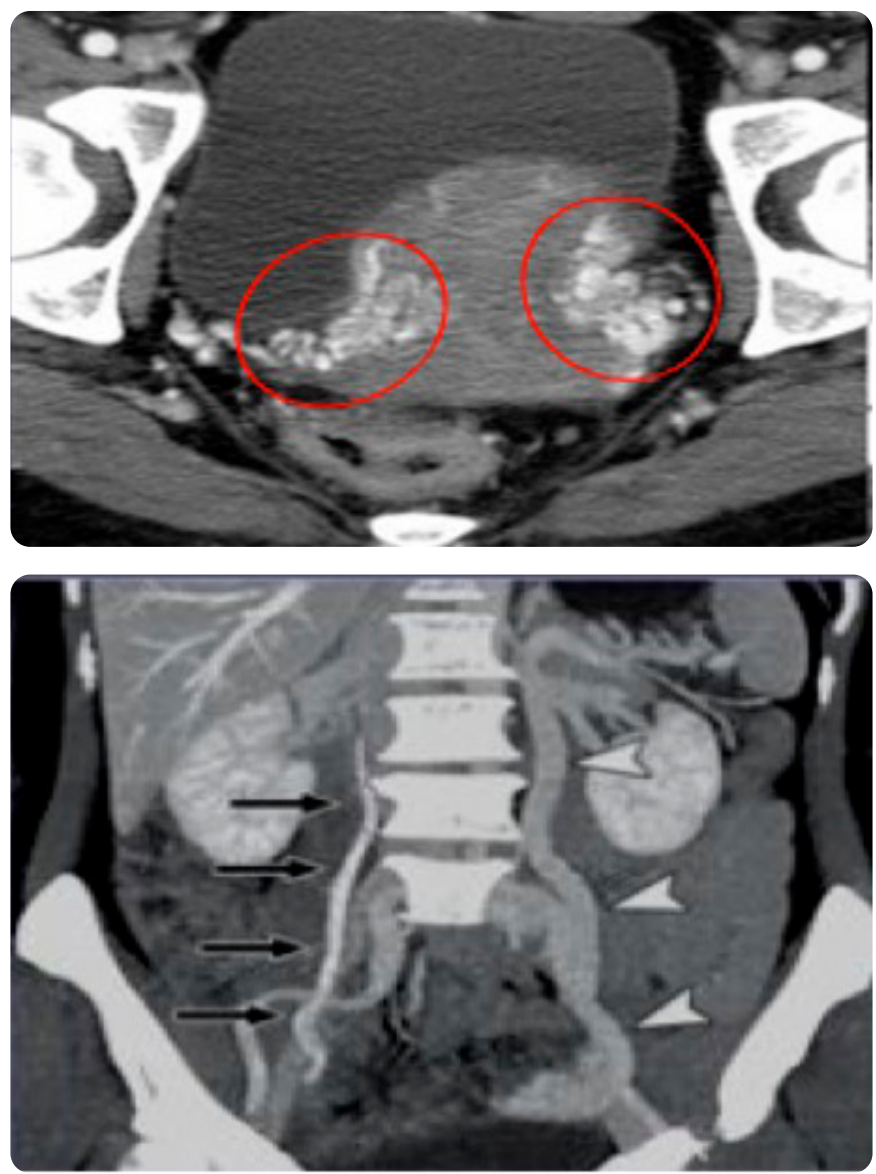

Figura 5. Venografía por TAC con medio de contraste en cortes axial y coronal: venas gonadales tortuosas y dilatadas produciendo el síndrome de congestión vascular venosa pélvica.

*Las imágenes 1, 4 y 5 son fotografías propias de los autores. Las imágenes 2 y 3 fueron proporcionadas por los proveedores directos de los dispositivos. 


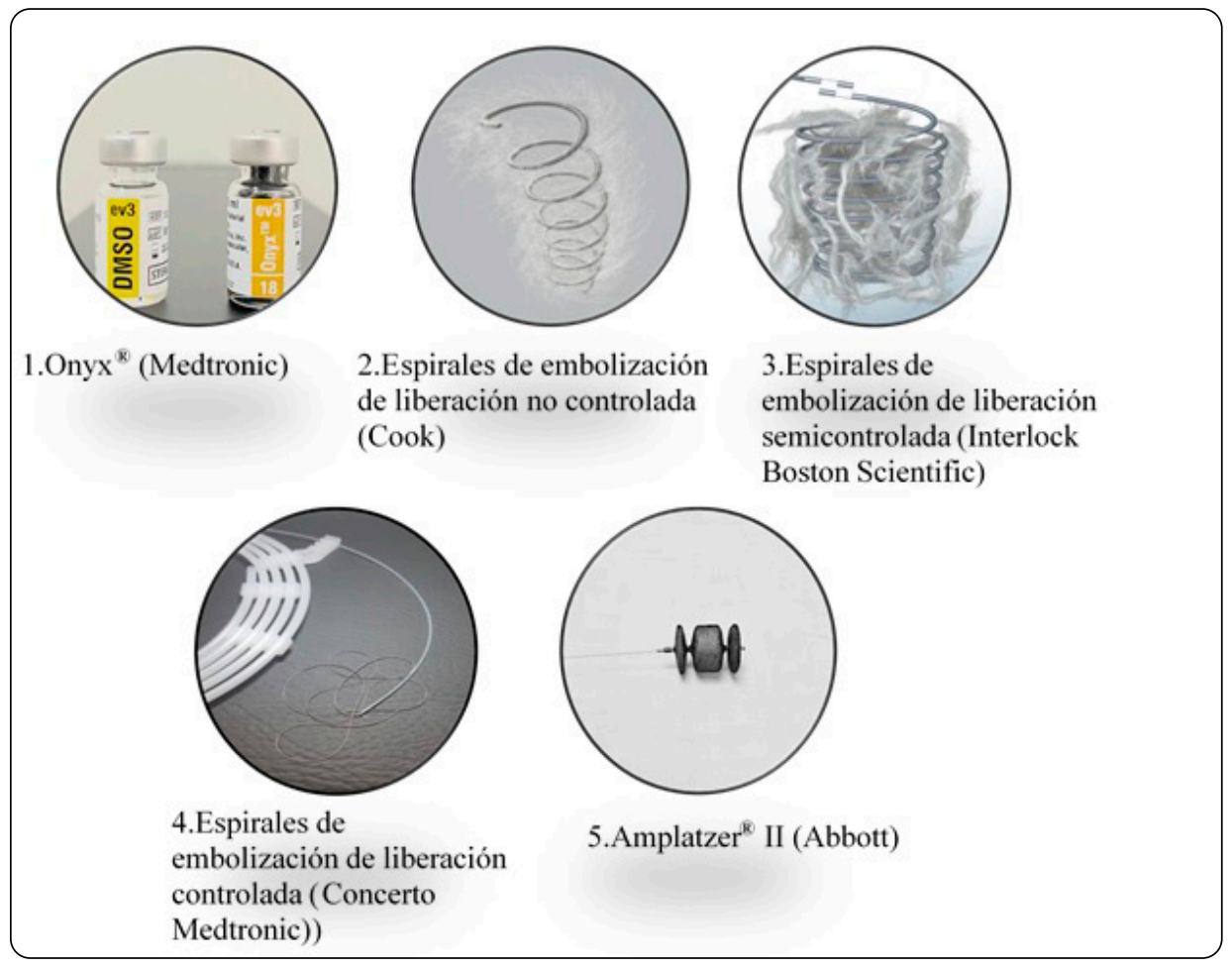

Figura 6. Diferentes métodos utilizados para manejo endovascular del SCP.

En la tabla 1 se resumen los criterios diagnósticos de cada ayuda imagenológica.

Tabla 1. Criterios diagnósticos de las modalidades imagenológicas en síndrome de congestión pélvica

\begin{tabular}{|c|c|}
\hline Ayuda imagenológica & Hallazgos \\
\hline Venografía convencional & $\begin{array}{l}\text { Arcadas venosas gonadales, uterinas y útero-ováricas dilatadas. } \\
\text { Diámetro }>5 \mathrm{~mm} \text {. } \\
\text { Flujo caudal retrógrado en la vena gonadal (unilateral o bilateral). } \\
\text { Llenado de las venas pélvicas pasando la línea media a través de la arcada útero-ovárica. } \\
\text { Opacificación de várices vulvovaginales o de los muslos. } \\
\text { Estancamiento del medio de contraste en las venas pélvicas. }\end{array}$ \\
\hline Ecografía transvaginal & $\begin{array}{l}\text { Várices parauterinas dilatadas múltiples. } \\
\text { Diámetro }>4 \mathrm{~mm} \text {. } \\
\text { Flujo lento }<3 \mathrm{~cm} / \mathrm{seg} \text {. } \\
\text { Vena arcuata dilatada en el miometrio que cruza la línea media. Configuración poliquística de los ovarios. }\end{array}$ \\
\hline $\begin{array}{l}\text { Ecografía } \\
\text { transabdominal }\end{array}$ & $\begin{array}{l}\text { Flujo retrógrado en la vena gonadal derecha o izquierda dilatada. } \\
\text { Vena gonadal dilatada }>5 \mathrm{~mm} \text {. }\end{array}$ \\
\hline Resonancia magnética & $\begin{array}{l}\text { Flujo caudal retrógrado del medio de contraste en la TR-MRA. } \\
\text { Várices parauterinas dilatadas. } \\
\text { Evidencia de lentitud en el flujo (heterogéneo o hiperintenso en T2). } \\
\text { Presencia de vena arcuata que cruza la línea media, las várices vulvares o los muslos } \\
\text { Configuración poliquística de los ovarios. } \\
\text { Ausencia de compresión estructural por alteración anatómica o masa obstructora. } \\
\text { Sin evidencia de endometriosis. }\end{array}$ \\
\hline $\begin{array}{l}\text { Tomografía } \\
\text { computarizada }\end{array}$ & $\begin{array}{l}\text { Cuatro venas parauterinas ipsilaterales tortuosas y dilatadas (mínimo una mayor a } 4 \text { mm). } \\
\text { Vena gonadal dilatada con un diámetro mayor a } 8 \text { mm unilateral o bilateral. } \\
\text { Ausencia de compresión estructural por alteración anatómica o masa obstructora. }\end{array}$ \\
\hline
\end{tabular}

Fuente: Adaptado de Bookwalter et al. (5). 


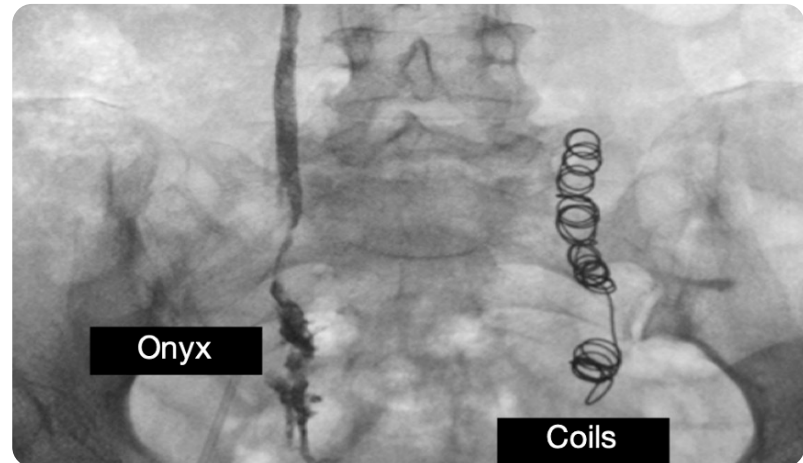

Figura 7. Venografía en paciente que inicialmente fue tratado por PCS mediante espirales de embolización en la vena gonadal izquierda y posteriormente con Ony ${ }^{\circledR}$ (Medtronic) en la vena gonadal derecha.
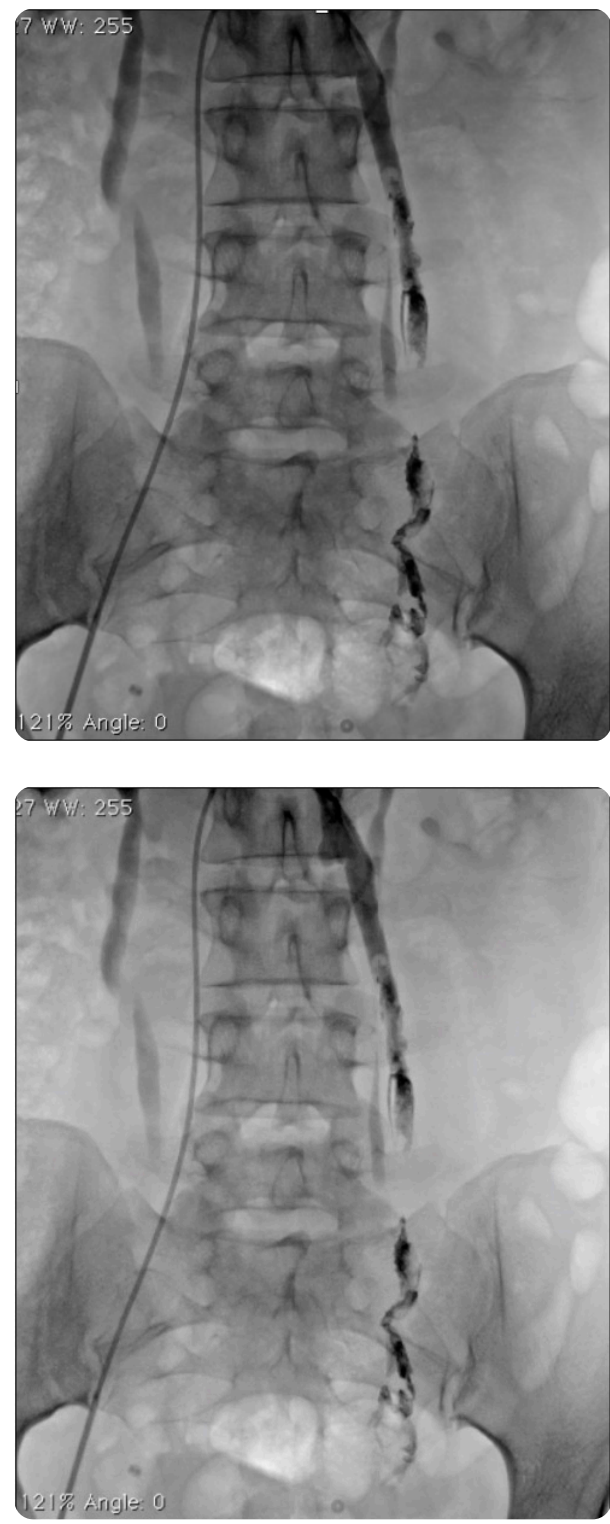

Figura 8. a y b) Venografía posterior a embolización selectiva con Onyx (Medtronic): adecuada competencia venosa posterior al manejo. La embolización debe iniciar por debajo de la articulación sacroilíaca inferior y concluir por encima de la articulación sacroilíaca superior.

\section{Tratamiento}

Para el tratamiento del SCP hay tres opciones de manejo: farmacológico, quirúrgico y endovascular, con embolización selectiva. Dentro de las alternativas en el manejo médico se encuentran el acetato de medroxiprogesterona, análogos GnRH, flebotónicos y AINES. Los principales efectos adversos asociados incluyen ganancia de peso, edema, sudoración nocturna y reaparición del dolor al suspender el tratamiento. El manejo quirúrgico cuenta con dos procedimientos: ooforectomía bilateral con o sin histerectomía, y la ligadura transperitoneal de las venas ováricas. Las posibles complicaciones asociadas a dichos procedimientos son: trombosis venosa profunda, hematoma retroperitoneal, íleo paralítico, íleo mecánico; además, se asocia con mayores estancias hospitalarias y mayor tiempo de recuperación. El manejo endovascular con embolización ha demostrado tener mayores tasas de éxito que el manejo farmacológico y quirúrgico $(4,5,25)$.

La terapia endovascular por medio de embolización fue descrita inicialmente por Edwards et al. en 1993 (26) y desde ese momento, mediante múltiples investigaciones, se ha establecido como el pilar en el manejo del PCS debido a su naturaleza mínimamente invasiva, morbilidad baja, recuperación corta, pocos días de hospitalización, mejoría del dolor, baja tasa de recurrencia del mismo y de complicaciones $(3,13,20,27-31)$ (figura 6).

Las principales complicaciones asociadas a la embolización son: migración de espirales de embolización a la circulación pulmonar, al arco venoso de la ilíaca externa o a la vena renal, perforación venosa, hematomas del sitio de punción y fiebre posterior al procedimiento $(4,27)$. Sin embargo, como se mencionó, la tasa de complicaciones es baja $(0-4 \%)(5,18,32)$.

Técnicamente, el procedimiento se puede llevar a cabo utilizando diferentes materiales como agentes esclerosantes, espirales de embolización, plugs tipo Amplatzer ${ }^{\circledR}$ (Abbott) (33) o agentes embólicos líquidos, como el Onyx ${ }^{\circledR}$ (Medtronic) (figura 7).

Un ensayo clínico publicado en 2013, en el que se les hizo seguimiento durante 5 años a 202 pacientes que recibieron terapia de embolización por dolor pélvico crónico secundario a SCP, documentó un éxito técnico del $100 \%$ y una resolución completa de los síntomas en el 34 \%, así como destacada mejoría del dolor y calidad de vida. Además, un bajo porcentaje $(1,9 \%)$ de complicaciones mayores, debidos a migración de espirales de embolización, sin importancia clínica posterior (25).

Por otra parte, en un estudio retrospectivo publicado en 2019, en el cual se analizaron 17 pacientes que fueron sometidas a embolización selectiva utilizando espirales de embolización en las venas ováricas, pélvicas o ambas, y luego a resección de paquetes varicosos vulvares o de muslos, se obtuvo un $100 \%$ de éxito técnico, con una media de un día de hospitalización, una mediana de 4 espirales de embolización utilizados en el procedimiento, notable mejoría en el dolor y en la calidad de vida y sin recurrencia del dolor durante 32 meses de seguimiento (3).

Las principales ventajas del Onyx ${ }^{\circledR}$ (Medtronic) vs. espirales de embolización son: oclusión inmediata, menos tiempo de reposo por parte de la paciente después del procedimiento para ser dada de alta, penetración a vasos colaterales. Desventaja: dolor en el momento de su colocación (figura 8).

Ventajas de espirales de embolización vs. Onyx ${ }^{\circledR}$ (Medtronic): No hay dolor en el momento de su implantación y adecuado efecto oclusor en el vaso. Desventajas: Riesgo de embolización paradójica a la circulación pulmonar y la paciente debe guardar reposo más estricto que con el Onyx ${ }^{\circledR}$ (Medtronic). 
El procedimiento de embolización de SCP puede demorar entre 15 y 90 minutos; depende, sin lugar a duda, de la experticia y habilidad del operador, la cantidad de venas insuficientes por ocluir y los materiales de embolización que se utilicen.

La recuperación de las pacientes usualmente es de 4 horas para las que reciben espirales de embolización y Amplatzer ${ }^{\mathbb{B}}$ (Abbott), y de una hora para las que se tratan con Onyx ${ }^{\circledR}$ (Medtronic).

Las reintervenciones postembolización son escasas y dependerán de que la paciente haya quedado bien embolizada en el momento del procedimiento, teniendo en cuenta la adecuada valoración e identificación de duplicaciones de las venas gonadales y variantes anatómicas, para lo cual se requiere un muy buen conocimiento de la anatomía.

En cuanto a los costos, el valor de dos espirales de embolización es equivalente a lo que cuesta un Amplatzer ${ }^{\circledR}$ (Abbott) y una ampolla de Onyx $^{\circledR}$ (Medtronic), aclarando que la variabilidad en su valor depende de la negociación de cada institución clínica con la industria.

\section{Conclusiones}

En conclusión, el SCP es una entidad altamente dolorosa, incapacitante e importante, y se debe tener en cuenta por su alta prevalencia y morbilidad asociadas. El correcto diagnóstico permite una intervención temprana enfocado hacia la mejoría y la resolución de los síntomas. El tratamiento endovascular ha demostrado ser una opción altamente confiable, segura, con bajas tasas de complicaciones, menor tiempo de recuperación y estancias hospitalarias más cortas en comparación con la opción quirúrgica; es relevante conocer el material y utilizarlo de manera adecuada, lo cual ayudará a evitar complicaciones.

\section{Referencias}

1. Hansrani V, Abbas A, Bhandari S, Caress AL, Seif M, McCollum CN. Trans-venous occlusion of incompetent pelvic veins for chronic pelvic pain in women: a systematic review. Eur J Obstet Gynecol Reprod Biol. 2015;185:156-63. doi:10.1016/j. ejogrb.2014.12.011

2. Brown CL, Rizer M, Alexander R, Sharpe EE 3rd, Rochon PJ. Pelvic congestion syndrome: systematic review of treatment success. Semin Intervent Radiol. 2018;35(1):3540. doi:10.1055/s-0038-1636519

3. Drazic BO, Zárate BC, Valdés EF, et al. Dolor pélvico crónico secundario a síndrome de congestión pélvica. Resultados del tratamiento endovascular de la insuficiencia venosa pelviana y várices genitales [Embolization of insufficient pelvic veins for pelvic congestion syndrome. Analysis of 17 cases]. Rev Med Chil. 2019;147(1):41-6. doi:10.4067/S0034-98872019000100041

4. O'Brien MT, Gillespie DL. Diagnosis and treatment of the pelvic congestion syndrome. J Vasc Surg Venous Lymphat Disord. 2015;3(1):96-106. doi:10.1016/j. jvsv.2014.05.007

5. Bookwalter CA, VanBuren WM, Neisen MJ, Bjarnason H. Imaging appearance and nonsurgical management of pelvic venous congestion syndrome. Radiographics. 2019;39(2):596-608. doi:10.1148/rg.2019180159

6. French S, Daugherty S, Thul S. Pelvic venous disease: identifying this commonly overlooked cause of chronic pelvic pain in women. J Nurse Pract. 2020;16(5):338-43. https://doi.org/10.1016/j.nurpra.2020.02.007.

7. Riding DM, Hansrani V, McCollum C. Pelvic vein incompetence: clinical perspectives. Vasc Health Risk Manag. 2017;13:439-47. doi:10.2147/VHRM.S132827

8. Corrêa MP, Bianchini L, Saleh JN, Noel RS, Bajerski JC. Pelvic congestion syndrome and embolization of pelvic varicose veins. J Vasc Bras. 2019;18:e20190061. doi:10.1590/1677-5449.190061

9. Borghi C, Dell'Atti L. Pelvic congestion syndrome: the current state of the literature. Arch Gynecol Obstet. 2016;293(2):291-301. doi:10.1007/s00404-015-3895-7

10. Antignani PL, Lazarashvili Z, Monedero JL, et al. Diagnosis and treatment of pelvic congestion syndrome: UIP consensus document. Int Angiol. 2019;38(4):265-83. doi: $10.23736 / \mathrm{S} 0392-9590.19 .04237-8$

11. Taylor HC Jr. Vascular congestion and hyperemia; their effect on function and structure in the female reproductive organs; the clinical aspects of the congestion-fibrosis syndrome. Am J Obstet Gynecol. 1949;57(4):637-53. doi:10.1016/0002-9378(49)90704-8
12. Tropeano G, Di Stasi C, Amoroso S, Cina A, Scambia G. Ovarian vein incompetence: a potential cause of chronic pelvic pain in women. Eur J Obstet Gynecol Reprod Biol. 2008;139(2):215-21. doi:10.1016/j.ejogrb.2007.11.006

13. Freedman J, Ganeshan A, Crowe PM. Pelvic congestion syndrome: the role of interventional radiology in the treatment of chronic pelvic pain. Postgrad Med J. 2010;86(1022):704-10. doi:10.1136/pgmj.2010.099473

14. Ahlberg NE, Bartley O, Chidekel N. Right and left gonadal veins. An anatomical and statistical study. Acta Radiol Diagn (Stockh). 1966;4(6):593-601 doi:10.1177/028418516600400601

15. Avgerinos ED, McEnaney R, Chaer RA. Surgical and endovascular interventions for nutcracker syndrome. Semin Vasc Surg. 2013;26(4):170-7. doi:10.1053/j.semvascsurg.2014.06.014

16. Mathis BV, Miller JS, Lukens ML, Paluzzi MW. Pelvic congestion syndrome: a new approach to an unusual problem. Am Surg. 1995;61(11):1016-8.

17. Ashour MA, Soliman HE, Khougeer GA. Role of descending venography and endovenous embolization in treatment of females with lower extremity varicose veins, vulvar and posterior thigh varices. Saudi Med J. 2007;28(2):206-12.

18. Koo S, Fan CM. Pelvic congestion syndrome and pelvic varicosities. Tech Vasc Interv Radiol. 2014;17(2):90-5. doi:10.1053/j.tvir.2014.02.005

19. Nicholson T, Basile A. Pelvic congestion syndrome, who should we treat and how? Tech Vasc Interv Radiol. 2006;9(1):19-23. doi:10.1053/j.tvir.2006.08.005

20. Dorobisz TA, Garcarek JS, Kurcz J, et al. Diagnosis and treatment of pelvic congestion syndrome: Single-centre experiences. Adv Clin Exp Med. 2017;26(2):269-76. doi: $10.17219 / \mathrm{acem} / 68158$

21. Park SJ, Lim JW, Ko YT, et al. Diagnosis of pelvic congestion syndrome using transabdominal and transvaginal sonography. AJR Am J Roentgenol. 2004;182(3):683-8. doi:10.2214/ajr.182.3.1820683

22. Jurga-Karwacka A, Karwacki GM, Schoetzau A, Zech CJ, Heinzelmann-Schwarz $\mathrm{V}$, Schwab FD. A forgotten disease: Pelvic congestion syndrome as a cause of chronic lower abdominal pain. PLoS One. 2019;14(4):e0213834. doi:10.1371/journal. pone. 0213834

23. Yang DM, Kim HC, Nam DH, Jahng GH, Huh CY, Lim JW. Time-resolved MR angiography for detecting and grading ovarian venous reflux: comparison with conventional venography. Br J Radiol. 2012;85(1014):e117-22. doi:10.1259/bjr/79155839

24. Coakley FV, Varghese SL, Hricak H. CT and MRI of pelvic varices in women. J Comput Assist Tomogr. 1999;23(3):429-34. doi:10.1097/00004728-199905000-00018

25. Laborda A, Medrano J, de Blas I, Urtiaga I, Carnevale FC, de Gregorio MA. Endovascular treatment of pelvic congestion syndrome: visual analog scale (VAS) long-term follow-up clinical evaluation in 202 patients. Cardiovasc Intervent Radiol. 2013;36(4):1006-14. doi:10.1007/s00270-013-0586-2

26. Edwards RD, Robertson IR, MacLean AB, Hemingway AP. Case report: pelvic pain syndrome--successful treatment of a case by ovarian vein embolization. Clin Radiol. 1993;47(6):429-31. doi:10.1016/s0009-9260(05)81067-0

27. Almeida GR, Silvinato A, Simões RS, Buzzini RF, Bernardo WM. Pelvic congestion syndrome - treatment with pelvic varicose veins embolization. Rev Assoc Med Bras (1992). 2019;65(4):518-23. doi:10.1590/1806-9282.65.4.518

28. Castenmiller PH, de Leur K, de Jong TE, van der Laan L. Clinical results after coil embolization of the ovarian vein in patients with primary and recurrent lower-limb varices with respect to vulval varices. Phlebology. 2013;28(5):234-8. doi:10.1258/ phleb.2012.011117

29. Maleux G, Stockx L, Wilms G, Marchal G. Ovarian vein embolization for the treatment of pelvic congestion syndrome: long-term technical and clinical results. J Vasc Interv Radiol. 2000;11(7):859-64. doi:10.1016/s1051-0443(07)61801-6

30. Venbrux AC, Chang AH, Kim HS, et al. Pelvic congestion syndrome (pelvic venous incompetence): impact of ovarian and internal iliac vein embolotherapy on menstrual cycle and chronic pelvic pain. J Vasc Interv Radiol. 2002;13(2 Pt 1):171-8. doi:10.1016 s1051-0443(07)61935-6

31. Meissner MH, Gibson K. Clinical outcome after treatment of pelvic congestion syndrome: sense and nonsense. Phlebology. 2015;30(1 Suppl):73-80. doi: $10.1177 / 0268355514568067$

32. Chung MH, Huh CY. Comparison of treatments for pelvic congestion syndrome. Tohoku J Exp Med. 2003;201(3):131-8. doi:10.1620/tjem.201.131

33. Basile A, Marletta G, Tsetis D, Patti MT. The Amplatzer vascular plug also for ovarian vein embolization. Cardiovasc Intervent Radiol. 2008;31(2):446-7. doi:10.1007/ s00270-007-9235-y

\section{Correspondencia}

Mateo Zapata Naranjo

Calle 48 FF Sur \# 42C-113

Medellín, Colombia

mateozn@gmail.com

Recibido para evaluación: 28 de diciembre de 2020

Aceptado para publicación: 25 de febrero de 2021 